\title{
GALILÉE-1: a validation and processing system for ENDF-6 and GND evaluations
}

\author{
Mireille COSTE-DELCLAUX ${ }^{1 a}$, Cédric JOUANNE ${ }^{1}$, Frédéric MOREAU ${ }^{1}$ and Claude MOUNIER ${ }^{1}$ \\ ${ }^{1}$ CEA Saclay, DEN, DM2S, SERMA, LLPR, F-91191 Gif-sur-Yvette, France
}

\begin{abstract}
GALILÉE-1 is the new validation and processing system for evaluated data, developed at CEA. This system can handle evaluations stored either in the ENDF-6 format or in the new General Nuclear Data (GND) format. It consists of various components respectively dedicated to read/write the evaluated data whatever the format is, to diagnose inconsistencies in the evaluated data and to provide continuous-energy and multigroup data as well as probability tables for transport and depletion codes. All these components are written in $\mathrm{C}++$ language and share the same objects. This paper describes the state of progress of the various parts of the system and gives some illustrations.
\end{abstract}

\section{Introduction}

GALILÉE-1 system is part of a CEA global development program dedicated to fine modelling of nuclear systems. It is carried out in synergy with the evaluation code CONRAD [1], and with the new generation of reactor physics codes, APOLLO3 [2], TRIPOLI-4 [3] and MENDEL [4]. It can handle evaluations stored either in the ENDF-6 or in the new General Nuclear Data (GND) format.

GALILÉE-1 system consists of:

- $\quad$ processing modules providing continuous-energy data (CE), multigroup data (MG) as well as probability tables (PT),

- $\quad$ interface modules creating application libraries (from these processed data) for deterministic or Monte Carlo transport codes, shielding codes or depletion codes,

- $\quad$ an automatic chain for creating these libraries.

GALILÉE-1 system originality lays in its two complementary running ways: an integrated one (Fig. 1) with a convivial and automatic chain for creating consistent and validated libraries for the various application codes and an open way for providing application codes with processing tools (Doppler on the fly for instance in a Monte Carlo transport code).

At the present, only the processing modules are under development. Cross-comparisons with other processing systems are systematically carried out at each step in order to fully master possible discrepancies.

\footnotetext{
${ }^{a}$ Corresponding author: mireille.coste-delclaux@cea.fr
} 


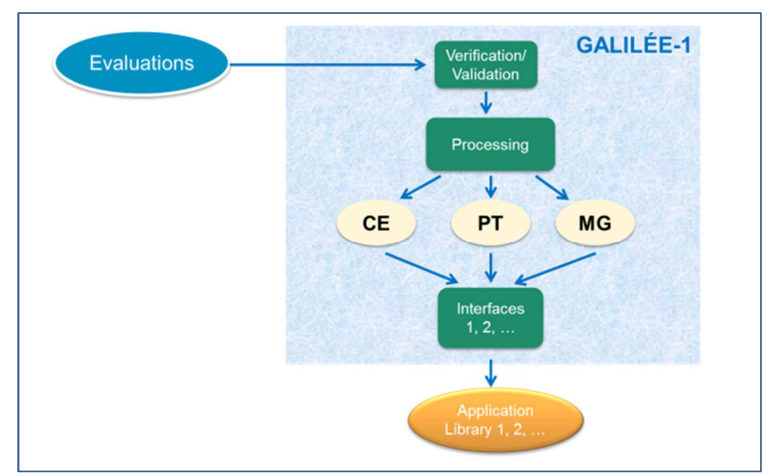

Figure 1. GALILÉE-1 Integrated running way.

\section{GALILÉE-1 processing modules}

Three main processing modules are under development in GALILÉE-1 system, a parser dedicated to read and write evaluated and processed data in ENDF-6 or GND format, a verification/validation tool described further and a processing code TREND (Treatment and Representation of Evaluated Nuclear Data). They are written in $\mathrm{C}++$ language and share common objects grouped in GBASE component. GBASE objects are completely independent from the input data (evaluation) format and the output data (processed data) format.

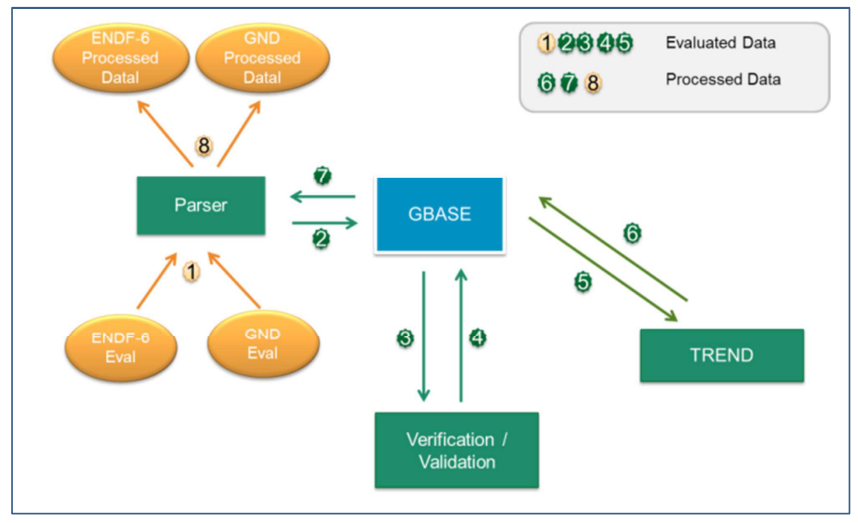

Figure 2. GALILÉE-1 Processing modules.

As shown in Figure 2, GBASE objects are initialized thanks to the Parser that reads the evaluation data. These objects are checked and eventually corrected by the Verification/Validation Tool and then processed data are created by TREND code. The objects storing processed data are also kept in GBASE and can be written on binary or character files by the Parser.

\section{GBASE objects}

The GBASE object hierarchy is very close to the GND object hierarchy. For each nucleus or element, we create a data base allowing us to store, in the same object, structure data and interaction data for a given projectile. GBASE structure data contain all the information needed to verify and eventually correct the evaluated data: masses, level scheme, spins, energy, half-life, decay modes, etc. GBASE interaction data contain the list of products that can be created by the interaction, all the information given by an evaluation (JEFF-3.2, ENDF/B-VII.1, JENDL4.0,...) but organized in such a way that processing is easier and processed data (CE data, PT, MG data, ...). 
Several GBASE structure data or several GBASE interaction data may exist in the same data base.

\section{Parser}

The Parser reads all the files of an evaluation in ENDF-6 format, and creates the GBASE objects corresponding to the resonance parameters, the reactions, and the kinematics of reaction products. Other GBASE objects are under construction. The reading of GND evaluations is under progress. Writing is not yet available.

\section{Verification / Validation}

One of the goals of GALILÉE-1 system is to test the consistency and the validity of nuclear data evaluations. We plan to perform a complete assessment of evaluated files before any treatment. Some checks can be performed by comparing data with the ones contained in structure databases, e.g. NUBASE or ENSDF. This is the case for:

- the masses of the nuclides, given in terms of neutron mass,

- the energies of the excited states reached in the inelastic scattering,

- gamma decay schemes of the excited states.

Some additional tests are designed to check the coherence between the data given in an evaluation:

- coherence between thresholds considered for various data of the same reaction,

- energy balance for reaction products,

- $\operatorname{spin} /$ parity of resonance parameters,

- normalization of distributions.

In this section, we present a list of test already considered in our code system allowing us to diagnose a list of incoherent values in an evaluation.

\subsection{Target mass and Q-value reaction}

The target mass is very important to determine the Q-value and the threshold of a reaction. In JEFF3.2 library, the $\mathrm{H} 2$ evaluated mass, in neutron mass, is 1.995712 instead of 1.9968 (underestimation of $1 \mathrm{MeV})$. This discrepancy should impact radiative capture Q-value but it is not the case, the correct value being given in the evaluation. Other examples of important nucleus mass discrepancies can be found in JEFF-3.2 evaluations: for example, more than 4.5 MeV for Xe134 and $366 \mathrm{keV}$ for Ti46.

In the case of transfer reactions, one can observe Q-value discrepancies higher than $1 \mathrm{MeV}$. Moreover, those values are not systematically given.

\subsection{Resonance state spin ( $\mathrm{J}$ value)}

The spin of a resonance state, $J$, is calculated from the orbital angular momentum $l$, the neutron spin and the target spin $I$. For Bk247 which spin is 3/2, in agreement with Nubase, the $J$ value given in JEFF-3.2 for $s$ resonances $(l=0)$, is $3 / 2$, out of the range of possible values, 1 or 2 . This discrepancy has a significant impact on the calculated cross-section due to the spin statistical multiplicative factor.

\subsection{Energy balance for emitted particles}

Evaluations contain energetic distributions for emitted particles at various incident energies. From these distributions, we can reconstruct total and partial average energies and compare them to available energy for the reaction. Interpolation schemes for emitted energy distributions and for incident energies play an important role to determine energy release. By analyzing these distributions, we are able to highlight incoherent values. 


\subsection{Negative angular distribution}

Angular distribution of emitted particles can be represented by Legendre polynomial expansion coefficients. This representation is particularly efficient in terms of compactness. However, the truncation at a given order can produce negative values for angular probabilities. Figure 3 deals with neutron elastic scattering on Au197 (for both JEFF-3.2 and ENDF/B-VII.1 libraries) at incident energy of $29 \mathrm{MeV}$. This is a difficult problem for Monte Carlo calculations. Indeed, the cumulative density function (CDF) is not continuously growing. In this case, for a sampling value, two or more scattering angles are possible.

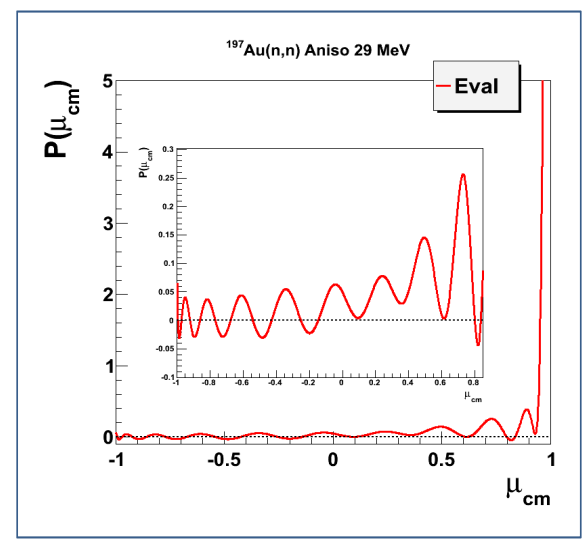

Figure 3. Angular distribution for ${ }^{197} \mathrm{Au}(\mathrm{n}, \mathrm{n})$ at $29 \mathrm{MeV}$.

\section{TREND}

TREND code aims at replacing NJOY99 and CALENDF codes in CEA application library production. It consists of three main parts, TREND_CE corresponding to NJOY RECONR, BROADR, UNRESR, THERMR and HEATR modules, TREND_PT corresponding to CALENDF treatment and TREND_MG corresponding to NJOY GROUPR module. At the present RECONR and TREND_PT functionalities are developed, BROADR/THERMR functionalities are under progress.

In order to validate TREND reconstruction at 0 Kelvin in the resolved resonance range, crosscomparisons with NJOY99 were carried out for all JEFF-3.2 nuclei $(\sim 470)$. In case of discrepancies, additional comparisons were made with NJOY2012 and PREPRO2015. About 25 nuclei show relative reconstruction discrepancies larger than 1.E-5, between NJOY99 and TREND, for MT1, MT2, MT102 and MT18 when it exists.

\subsection{Discrepancies on MT2}

For Fe54 and Os192, we observe relative discrepancies on MT2 (Table 1) not yet fully understood. For Fe54 these discrepancies appear between $3453.125 \mathrm{eV}$ and $3497.071 \mathrm{eV}$ and for Os192, between $2313.995 \mathrm{eV}$ and $2314.521 \mathrm{eV}$.

Table 1. Maximum relative discrepancies on MT2 between NJOY99 and TREND.

\begin{tabular}{|c|c|c|}
\hline & Maximum relative discrepancy & Energy (eV) \\
\hline Fe54 & $1.26 \mathrm{E}-3$ & 3475.009 \\
\hline Os192 & $2.01 \mathrm{E}-4$ & 2314.337 \\
\hline
\end{tabular}




\subsection{Discrepancies on MT102}

For $\mathrm{C} 13, \mathrm{Kr} 86, \mathrm{O} 17, \mathrm{O} 18, \mathrm{~Pb} 206, \mathrm{~Pb} 208, \mathrm{Sr} 88$ and $\mathrm{Sr} 90$, discrepancies occur on MT 102 reconstruction with, for all nuclei, the same "jump" between $0.01 \mathrm{eV}$ and $0.1 \mathrm{eV}$ (Fig.4). This problem disappears with NJOY2012 (except for O17 and O18) and with PREPRO15 for O18.

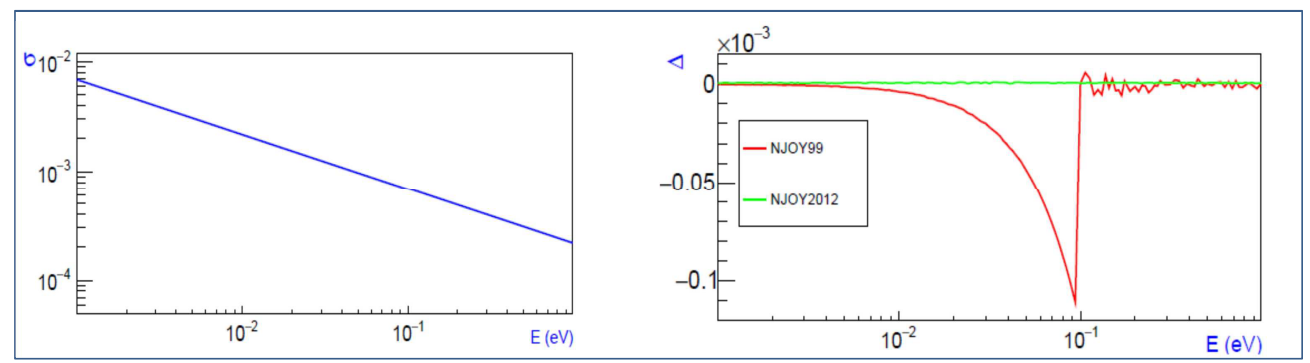

Figure 4. Relative discrepancies between NJOY and TREND on reconstructed MT102 file for C13.

\subsection{Discrepancies on MT1}

On other nuclei, discrepancies may appear on MT1, either when summing the background crosssections or because of threshold problems. TREND uses, for the reconstruction, the partial crosssections and not the redundant cross-sections. Moreover, as NJOY does, TREND modifies the threshold given in the evaluation when its value is not correct. But some differences remain.

As an example, for O16 in JEFF-3.2, the MT107 background file is not consistent with the sum of the MT800-MT803 files, above the threshold of the first excited state of C13 (MT801). That leads to discrepancies between NJOY 99 and TREND (Table 2). This problem is solved with NJOY2012.

Table 2. Maximum relative discrepancies on MT107 for O16.

\begin{tabular}{|c|c|c|}
\hline & Maximum relative discrepancy & Energy (eV) \\
\hline NJOY99/TREND & $8.96 \mathrm{E}-4$ & 5910000. \\
\hline NJOY2012/TREND & $4.84 \mathrm{E}-6$ & 2380000. \\
\hline
\end{tabular}

\section{Conclusion}

GALILÉE-1 system allows R\&D activity on new processing methods. It is also a powerful tool for nuclear data analysis. It will produce soon Monte Carlo transport libraries from validated evaluations, which is a first important step for our renewed library creation chain.

The authors acknowledge the Industrial Partners AREVA-NP and EDF for their partial financial support through SINET/DNT project.

\section{References}

1. P. Archier et al. "CONRAD evaluation code: Development status and Perspectives", Nuclear Data Sheets, 118, 488-490, (2014)

2. H. Golfier et al., "APOLLO3: a common project of CEA, AREVA and EDF for the development of a new deterministic multi-purpose code for core physics analysis," M\&C 2009, Saratoga Springs, New York, USA (2009)

3. E Brun et al., "Overview of TRIPOLI-4 version 7, Continuous-energy Monte Carlo Transport Code", ICAPP 2011, Nice, France (2011)

4. S. Lahaye et al., "First Verification and Validation Steps of MENDEL Release 1.0 Cycle Code System”, PHYSOR 2014, Kyoto, Japan, (2014) 
